\title{
Geschlechterstereotype in Persona- Beschreibungen
}

\author{
Nicola Marsden ${ }^{1}$, Jasmin Link ${ }^{2}$, Elisabeth Büllesfeld ${ }^{2}$ \\ Fakultät IT, Hochschule Heilbronn ${ }^{1}$ \\ Fraunhofer-Institut für Arbeitswirtschaft und Organisation IAO, Stuttgart ${ }^{2}$
}

\begin{abstract}
Im nutzungszentrierten Design werden Personas als zentraler Teil des Gestaltungsprozesses genutzt. Um zu untersuchen, welche Rolle Personas beim Fortschreiben bestehender Geschlechterstereotype spielen, wurden in einer empirischen Analyse 170 Persona-Beschreibungen hinsichtlich des dargestellten sozialen Umfelds, der Freizeitbeschäftigungen und der Technikkompetenz untersucht. Die Ergebnisse zeigen geschlechterstereotype Darstellungen auf der Dimension Wärme/Gemeinschaft. Diese werden diskutiert und es werden Empfehlungen für die Gestaltung mit Personas gegeben.
\end{abstract}

\section{Personas zur Repräsentation von Nutzenden}

Gendergerechtigkeit spielt in der Forschung zu Human-Computer Interaction eine immer wichtigere Rolle (Breslin \& Wadhwa, 2015; Grudin \& Williams, 2013). Dabei ist die Berücksichtigung von Nutzerinnen und Nutzern mit ihren diversen Lebenslagen zentral (Marsden \& Kempf, 2014). Die Persona-Methode ist eine Herangehensweise, die hier häufig als Werkzeug eingesetzt wird (Grudin, 2006; Nielsen, 2013; Pruitt \& Adlin, 2006). In der Persona-Methode werden fiktive Repräsentanten von Nutzerinnen und Nutzern geschaffen. Personas werden im Rahmen des Gestaltungsprozesses als prototypische Stakeholder genutzt, um eine aktive Auseinandersetzung mit den Nutzerinnen und Nutzern, die Kommunikation zwischen Gestaltungs- und Entwicklungsteam sowie zwischen Auftraggebenden und nehmenden zu unterstützen. Personas können im gesamten menschzentrierten Gestaltungsprozess eingesetzt werden und sowohl als formatives als auch als evaluatives Werkzeug eingesetzt werden (Nielsen, 2013).

Der Einsatz von Personas birgt die Gefahr, dass unreflektiert Stereotype zum Einsatz kommen - wobei in einem gewissem Maße Stereotype in der Arbeit mit Personas unumgänglich sind (Turner \& Turner, 2011). Die Gründe für die Anwendung von Stereotypen auf Personas sind vielfältig. In erster Linie handelt es sich hier um den Einsatz der I-Methodology (Bath, 2014a, 2014b; Oudshoorn, Rommes, \& Stienstra, 2004) bzw. des fundamentalen Gestal- 
tungsfehlers (Ritter, Baxter, \& Churchill, 2014): Die Entwickelnden gehen von sich selbst aus und attribuieren die eigenen Vorstellungen, Herangehensweisen, Ziele etc. auf die Personas und konzipieren diese dann entweder als ihnen ähnlicher oder - entsprechend dem psychologischen Kontrasteffekt - als unähnlicher, als sie de facto sind. Auch andere Faktoren wie Zeitmangel oder Gruppenzugehörigkeiten fördern einen Rückgriff auf Stereotype bei Gestaltung und Einsatz von Personas (Marsden, Link, \& Büllesfeld, 2014), ebenso eine wenig detaillierte Beschreibung von Personas (Nielsen, 2013).

Als Lösung zur Vermeidung bzw. Reduzierung von sterotypen Beschreibungen im menschzentrierten Gestaltungsprozess gilt es in erster Linie, mit echten Nutzerinnen und Nutzern zu arbeiten (Buchmüller, Joost, Bessing, \& Stein, 2011; Holtzblatt \& Beyer, 2015; Ritter et al., 2014). Darüber hinaus gibt es eine Reihe von Herangehensweisen, um problematische Vergeschlechtlichungen in der Human-Computer Interaction $\mathrm{zu}$ entdecken, $\mathrm{zu}$ thematisieren oder $\mathrm{zu}$ vermeiden werden, z.B. das „Gender Extended Research and Development“ (GERD)-Modell (Draude, Maaß, \& Wajda, 2014) Mind Scripting (Allhutter, 2012), geschlechter- und intersektionalitätskritische Softwaregestaltung (Paulitz \& Prietl, 2014), Diffractive Design (Bath, 2014a; Ernst, 2014), das intersektionale „Sanduhr-Modell“ (Lucht, 2014), die verschiedenen Möglichkeiten feministischer Interventionen in den Gestaltungsprozess (Rommes, 2014), die Reflective/Reflexive Methods (Bardzell \& Churchill, 2011) etc. Einige Ansätze der Vermeidung von Geschlechterstereotypen fokussieren spezifisch auf den Einsatz von Personas (Marsden et al., 2014), nutzen Personas, um eine Reflexion von Geschlechterstereotypen zu unterstützen (Källhammer \& Nilsson, 2012) oder gezielt dafür, die Anliegen beider Geschlechter im Gestaltungprozess von Software zu berücksichtigen (Burnett et al., 2014).

Um ein weitergehendes Verständnis davon zu bekommen, welche Rolle Geschlechterstereotype in der Arbeit mit Personas spielen, wurde eine Untersuchung von im Einsatz befindlichen Personas durchgeführt. Im Folgenden wird zunächst der theoretische Hintergrund von Geschlechterstereotypen skizziert, dann wird die Untersuchung beschrieben, die Ergebnisse werden vorgestellt und diskutiert und Implikationen für den Gestaltungsprozess abgeleitet.

\section{Geschlechterstereotype}

Geschlechterstereotype beinhalten Wissen darüber, welche Eigenschaften und Verhaltensweisen für Männer und Frauen ein einer Gesellschaft bezeichnend sind und bei ihnen vermeintlich zu beobachten sind (Kite, Deaux, \& Haines, 2008). Als soziokognitive Strukturen haben Stereotype eine duale Struktur dahingehend, dass es sich einerseits um individuelles Wissen der jeweiligen Person handelt, andererseits um sozial geteiltes Wissen, das durch Kultur und zwischenmenschlichen Konsens hergestellt und verfestigt wird (Eckes, 2008). Die kognitive Komponente (der Stereotyp im engeren Sinne) geht einher mit einer emotionalen Komponente und einer Verhaltenskomponente. Der Dreiklang von geschlechtsbezogenen Stereotypen, Affekten und Verhaltensweisen, die in einen ungleichen sozialen Status von Männern und Frauen resultieren, wird als Sexismus bezeichnet (Swim \& Campbell, 2003). Geschlechterstereotype sind häufig nicht bewusst und werden automatisch und ohne aktives 
Zutun angewendet (Banaji \& Hardin, 1996), d.h. sie entfalten ihre Wirksamkeit oft in Form von impliziten Assoziationen (Greenwald, McGhee, \& Schwartz, 1998).

Durch die duale Struktur von Geschlechterstereotypen ist es so, dass durch jeden Akt der Stereotypisierung der Stereotyp erneut konsensuell validiert, d.h. das vermeintliche Wissen über für Männer oder Frauen typische Eigenschaften und Verhaltensweisen wiederum fortgeschrieben wird. Hinsichtlich der Inhalte von Geschlechterstereotypen zeichnet die Forschung ein klares Bild: Frauen werden Eigenschaften zugesprochen, die eher auf der Dimension Wärme, Soziales oder Gemeinschaftsorientierung angeordnet sind. Merkmale, die häufiger Männern zugeordnet werden, sind auf der Dimension der aufgabenbezogenen Kompetenz, Instrumentalität oder Selbstbehauptung zu verorten (Fiske, Cuddy, \& Glick, 2007), ein Ergebnis, welches interkulturell und auch für Deutschland bestätigt wird (Ebert, Steffens, \& Kroth, 2014; Eckes, 2002). Neben diesen Globalstereotypen von Männern und Frauen gibt es Substereotype, d.h. Globalstereotype sind strukturell heterogen und setzen sich aus einer Reihe in sich homogener Kategorien zusammen. So werden zum Beispiel arbeitende Mütter und arbeitende Väter sehr unterschiedlich wahrgenommen: Arbeitende Mütter werden als kompetenter eingestuft, verlieren aber auf der Dimension Wärme, die mit Mütter und Hausfrauen verbunden ist. Arbeitende Väter hingegen werden als eine erfolgreiche Kombination in den Dimensionen Wärme und Kompetenz wahrgenommen (Cuddy, Fiske, \& Glick, 2004).

Für die Globalstereotype, mit denen Frauen eher Eigenschaften der Dimension Wärme/Gemeinschaftsorientierung und Männer eher Kompetenz/Instrumentalität zugeschrieben wird, hat sich gezeigt, dass sie über die Zeit sehr stabil sind. Allerdings hat sich in den letzten Dekaden die von Frauen über sich selbst berichtete Instrumentalität kontinuierlich erhöht - die selbstberichtete Expressivität von Männern bleibt dabei gleichzeitig unverändert (Twenge, 1997, 2009). Für die Globalstereotype gibt es zwei Erklärungsansätze: Die Theorie der sozialen Rollen (Eagly, Wood, \& Diekman, 2000) zeigt auf, dass Menschen davon ausgehen, dass Frauen und Männer diejenigen Merkmale aufweisen, die für ihre jeweiligen sozialen Rollen, insbesondere für ihre Familien- und Berufsrollen, typisch sind. Menschen verallgemeinern dann vom beobachteten Rollenverhalten unmittelbar auf Eigenschaften der Rolleninhabenden und vernachlässigen dabei den Einfluss der verhaltenswirksamen Rollenanforderungen. Ergänzend werden die Globalstereotype durch das Stereotypinhaltsmodell erklärt (Fiske, Cuddy, Glick, \& Xu, 2002). Es besagt unter anderem, dass der Status einer Gruppe die Einordnung auf der Kompetenzdimension bestimmt, und zwar dahingehend, dass Gruppen mit hohem Status als kompetent eingeschätzt werden. Die Einordnung einer Gruppe auf der Wärmedimension dagegen wird durch die Art der Interdependenz bestimmt, und zwar so, dass kooperative Gruppen als warm bzw. als unbedrohlich für die eigenen Gruppenziele und kompetitive Gruppen als kalt bzw. als bedrohlich eingeschätzt werden.

Es gibt eine Vielzahl von Methoden zur Messung von Stereotypen (Eckes, 2008; Kite et al., 2008). Um medial vermittelte stereotype Darstellungen der Geschlechter zu untersuchen, werden meist inhaltsanalytische Verfahren angewendet (Collins, 2011; Rudy, Popova, \& Linz, 2011), sie zeigen, dass Frauen in der medialen Vermittlung eher unterrepräsentiert sind, untergeordnet, in stereotyp femininen Rollen oder negativ dargestellt werden (Collins, 2011). Bisher wurden Persona-Beschreibungen noch nicht als mögliche Kristallisationspunkte von Geschlechterstereotypen in den Blick genommen. Diese Forschungslücke sollte mit 
der vorliegenden Untersuchung geschlossen werden, vor allem vor dem Hintergrund dass eine geschlechterstereotype Darstellung an dieser Stelle den gesamten Gestaltungsprozess beeinflussen kann. Eine Forschungsfrage lautet entsprechend, welche Hinweise existierende Persona-Beschreibungen auf Geschlechterstereotype geben - die hier berichteten Ergebnisse fokussieren sich dabei auf das soziale Umfeld, die Freizeitbeschäftigungen und die Technikkompetenz der Personas.

\section{Methode}

Als Stichprobe wurden insgesamt 170 Persona-Beschreibungen analysiert, dabei handelte es sich um 73 Beschreibungen weiblicher, und 83 Beschreibungen männlicher Personas, 5 Beschreibungen von Paaren, sowie 9 Beschreibungen, die mit ,weiblich, ggf. männlich“ oder „Familie XY“ keine Zuordnung zulassen. Um eine möglichst breite Stichprobe zur Auswertung existierender Personas zu erhalten, wurde eine Recherche öffentlich zugänglicher Personas (z.B. über Projekthomepages) durchgeführt, darüber hinaus gab es im November 2014 einen Aufruf, im Einsatz befindliche Personas zur Verfügung zu stellen. Bei dem Aufruf wurde nicht erwähnt, dass es um eine Auswertung zum Thema Gender ging, um Effekte der sozialen Erwünschtheit dahingehend zu vermeiden. Dass Wirtschaftsunternehmen hier mit entsprechenden Informationen deutlich restriktiver sind als öffentliche Auftraggebende schlägt sich auch in der Stichprobe nieder: Von den 21 Persona-Sets stammen sieben aus Quellen von öffentlicher Hand oder Non-Profit-Organisationen (50 Persona-Beschreibungen, kurz PB), sechs aus EU-Projekten (73 PB), drei von Universitäten (13 PB) und fünf von Unternehmen aus der Wirtschaft (34 PB). Die 21 Persona-Sets hatten zwischen 2 und 30, meist zwischen 5 und 10, und durchschnittlich 8 Personas pro Set. Neun der Sets liegen in deutscher Sprache vor (51 PB), eines in Niederländisch (3 PB), und elf in Englisch (116). Die beschriebenen Personas leben in Deutschland (60), Österreich (10), den USA (30), Großbritannien (12), Schweden (6) Australien (5) und anderen europäischen Ländern. Anwendungsdomäne der Persona-Sets waren IT-Systeme in öffentlich nutzbaren Einrichtungen wie Bibliotheken, Universitäten und Museen (sechs Mal), IT-Systeme im Wohn- und Lebensumfeld (fünf Mal), zudem ging es um Assistenz-Technologien, Systeme aus dem Verkehrsbereich und um konkrete Produkte oder Dienstleistungen.

Das Vorgehen sah folgendermaßen aus: Die Persona-Beschreibungen wurden nach theoriegeleitet festgelegten Attributen inhaltsanalytisch ausgewertet (Früh, 2011). Als Untersuchungseinheit wurden die einzelnen Personas gewählt. Basis der hier dargestellten Auswertung waren folgende theoriegeleitete Attribute: soziales Umfeld, Freizeitbeschäftigungen und Technikaffinität. Die Analyse erfolge in zwei Phasen.

In der ersten Phase wurde das Kategoriensystem operationalisiert und die in den PersonaBeschreibungen identifizierten Hinweise auf die Attribute wurden in einer ersten Durchsicht aller Persona-Beschreibungen als Ausprägung (z.B. für Kinder: „,nicht erwähnt“, ,kinderlos“, „eine Tochter“ etc.) oder als Skala operationalisiert. Die entwickelte Skala ordnet den Dimensionen einen Wert zwischen null und fünf für die Wichtigkeit der Dimension innerhalb einer Persona-Beschreibung zu. Um diese Einschätzung möglichst objektiv und nachvoll- 
ziehbar zu machen, wurden die Zwischenwerte beschrieben, und die Kriterien für Werte festgelegt. Teilweise wurden auch Paare, Familien oder Geschwister als PersonaBeschreibungen im selben Format wie Einzelpersonen beschrieben. In diesem Fall wurden die dargestellten Personen als Entität erfasst, in der Codierung wurde dann zum Beispiel bei einem Paar der Mittelwert als Alter angegeben, als Geschlecht wurde „Paar“ codiert.

In der zweiten Analysephase wurden die auf dieser Basis entwickelten Ausprägungen der Kategorien und Skalen frequenzorientiert ausgewertet und entsprechend der Forschungsfrage wurden die Attribute soziales Umfeld, Freizeitbeschäftigungen und Technikaffinität jeweils im Hinblick auf das Geschlecht der Persona betrachtet.

\section{Ergebnisse}

Insgesamt zeigte sich, dass sich die Persona-Beschreibungen in Umfang und Inhalten erheblich unterschieden: Neben tabellarischen Auflistungen in der Art eines Persona-Posters waren narrative Beschreibungen zu finden, die sich zwischen wenigen Zeilen Text plus Bild und dreiseitigen Texten bewegten, in denen Ziele, Motivationen und weitere Hintergründe aus dem Leben der Personas dargestellt wurden. Im folgenden werden die Ergebnisse der zweiten Analysephase für die Bereiche soziales Umfeld, Freizeitbeschäftigungen und Technikaffinität dargestellt. Wie oben dargestellt wurde frequenzorientiert für die Untersuchungseinheit „Persona“ ausgewertet.

\subsection{Soziales Umfeld}

Die Analyse der Persona-Beschreibungen hinsichtlich der Erwähnung von Kindern zeigte, dass für die Hälfte der weiblichen, jedoch nur einem Drittel der männlichen Personas Kinder erwähnt wurden. Das Geschlecht der Kinder war in den meisten Fällen nicht spezifiziert, in den Fällen, in denen hier eine Angabe vorhanden war hielt sich die Anzahl der Töchter und Söhne insgesamt die Waage. Enkelkinder gab es bei männlichen und weiblichen Personas zu gleichen Anteilen. Die explizite Erwähnung von Kinderlosigkeit war bei weiblichen Personas tendenziell häufiger. Insgesamt gab es so bei $63 \%$ der männlichen und $41 \%$ der weiblichen Personas keine Erwähnung von Kindern oder Enkelkindern.

\begin{tabular}{|l|r|r|r|}
\hline \multicolumn{1}{|c|}{ Kinder } & \multicolumn{1}{|c|}{ gesamt } & Persona weiblich & Persona männlich \\
\hline nicht erwähnt & $55 \%$ & $41 \%$ & $63 \%$ \\
\hline kinderlos & $7 \%$ & $8 \%$ & $5 \%$ \\
\hline $\begin{array}{l}\text { Kinder (Enkel } \\
\text { nicht erwähnt) }\end{array}$ & $27 \%$ & $40 \%$ & $22 \%$ \\
\hline Kinder und Enkel & $11 \%$ & $11 \%$ & $11 \%$ \\
\hline
\end{tabular}

Tabelle 1: Bezug auf Kinder den Persona-Beschreibungen $(N=170)$ 
Neben Integration von Kindern und Enkelkindern in die Persona-Beschreibung wurde auch analysiert, in welchem Ausmaß weitere Personen aus dem sozialen Umfeld der Persona (Partner_in, Vorgesetzte, Freunde, Geschwister, Eltern etc.) explizit erwähnt wurden. Die Persona-Beschreibungen unterschieden sich deutlich in der Nennung bzw. Nicht-Nennung solcher Nebenpersonas: Beinahe ein Drittel der männlichen Persona-Beschreibungen enthielten keine weiteren Verweise auf andere Personen, bei den weiblichen waren es knapp ein Sechstel, d.h. bei männlichen Persona-Beschreibungen wurde doppelt so häufig keine Aussage zum sozialen Umfeld gemacht wie bei weiblichen.

\subsection{Freizeitbeschäftigungen}

Von den 170 Persona-Beschreibungen enthielten 49 Informationen über Hobbies und Freizeitbeschäftigungen: Bei $29 \%$ der weiblichen und $34 \%$ der männlichen Personas wurden solche Aktivitäten erwähnt, bei den männlichen Personas wurden zudem tendenziell mehr Freizeitaktivitäten aufgeführt. Wie in Tabelle 1 dargestellt finden sich Aktivitäten häufig sowohl in den Beschreibungen männlicher als auch weiblicher Personas, jedoch gibt es auch eine Reihe von Aktivitäten, die ausschließlich bei den Persona-Beschreibungen eines Geschlechts zu finden sind. Dabei handelt es sich bei den Aktivitäten, die nur bei weiblichen Personas beschrieben sind, nur um Einzelnennungen, bei den männlichen PersonaBeschreibung gibt es mehrere Beschäftigungen, die bei weiblichen Persona-Beschreibungen gar nicht vorkommen, allen voran die Beschäftigung mit Technik mit sieben Nennungen.

\begin{tabular}{|c|c|}
\hline $\begin{array}{l}\text { Nur in Beschreibungen männli- } \\
\text { cher Personas genannt: }\end{array}$ & $\begin{array}{ll}\text { - } & \text { Technik (7 mal genannt) } \\
\text { - } & \text { Fußball, Freunde treffen (jeweils } 2 \text { mal genannt) } \\
\text { - } & \text { Angeln, Jagen, Poker, Schiffsmodelle bauen, Wein, } \\
& \text { Lokalgeschichte, Ahnenforschung, MMORPG, } \\
& \text { Computerspiele, Medien, Katze, Segeln, Golf, Ke- } \\
& \text { geln, Ausflüge, Radfahren (jeweils } 1 \text { mal genannt) }\end{array}$ \\
\hline $\begin{array}{l}\text { In Beschreibungen beider Ge- } \\
\text { schlechter genannt, aber häufi- } \\
\text { ger bei männlichen Personas: }\end{array}$ & $\begin{array}{ll}- & \text { Filme/Kino } \\
- & \text { Garten } \\
- & \text { Kochen } \\
- & \text { Wandern } \\
- & \text { Lesen }\end{array}$ \\
\hline $\begin{array}{l}\text { In Beschreibungen weiblicher } \\
\text { und männlicher Personas gleich } \\
\text { häufig genannt: }\end{array}$ & $\begin{array}{ll}- & \text { Reisen (jeweils } 4 \text { mal) } \\
- & \text { Ehrenamt, Parties/Feiern (jeweils } 1 \text { mal) }\end{array}$ \\
\hline $\begin{array}{l}\text { In Beschreibungen beider Ge- } \\
\text { schlechter genannt, aber häufi- } \\
\text { ger bei weiblichen Personas: }\end{array}$ & $\begin{array}{ll}- & \text { Musik } \\
\text { - } & \text { Fotos } \\
\text { - } & \text { Sport (unspezifisch, keine Sportart genannt) } \\
\text { - } & \text { Kultur (Kunst, Ballett, Museen, Galerien) }\end{array}$ \\
\hline $\begin{array}{l}\text { Nur in Beschreibungen weibli- } \\
\text { cher Personas genannt: }\end{array}$ & $\begin{array}{l}\text { - Stricken, Fernsehen, Jazz, Klavierspielen, Gitarre, } \\
\text { Tetris, MySpace, Schwimmen, Aerobic, Spiele- } \\
\text { abend (jeweils } 1 \text { mal genannt) }\end{array}$ \\
\hline
\end{tabular}

Tabelle 2: In den Persona-Beschreibungen genannte Freizeitbeschäftigungen 


\subsection{Technikkompetenz}

Die frequenzbasierte Auswertung der Persona-Beschreibungen zeigte keine Unterschiede in der Technikkompetenz: Die durch die Rater vorgenommene Einschätzung entlang der Skala Technikkompetenz ergibt für die Durchschnittswerte männlicher und weiblicher Personas keine Unterschiede. Auch wurde diese Dimension bei beiden Geschlechtern ähnlich oft nicht erwähnt. Die Daten deuten jedoch darauf hin, dass in den Personabeschreibungen sowohl eine große Begeisterung für Technik, als auch deren entschiedene Ablehnung eher bei männlichen Personas beschrieben wird.

\section{Diskussion}

Die frequenzanalytische Auswertung von 170 Persona-Beschreibungen hinsichtlich sozialem Umfeld, Freizeitbeschäftigungen und Technikkompetenz gibt Hinweise auf das Vorhandensein stereotyper Geschlechterdarstellungen in den untersuchten Personas.

Die in der Forschung zu Inhalten von Geschlechterstereotypen (Eckes, 2008) zentrale Verquickung von Frau-Sein mit der Dimension des Sozialen ist in den Daten deutlich erkennbar: Bei weiblichen Personas werden Kinder deutlich häufiger erwähnt als bei männlichen Personas. Die Erwähnung weiterer Personen im sozialen Umfeld der Persona ist bei männlichen Personas ebenfalls seltener. Auch die Ergebnisse zu den für die Personas dargestellten Freizeitbeschäftigungen zeigen geschlechterstereotype Züge: In den Beschreibungen der weiblichen Personas gab es außerhalb der für beide Geschlechter vorkommenden Freizeitbeschäftigungen Hobbys wie Stricken, Fernsehen oder Aerobic für weibliche Personas, bei den männlichen Personas waren es Technik, Fußball oder Schiffsmodelle.

In der dargestellten Untersuchung unterscheiden sich männliche und weibliche Personas nicht in ihrer Technikkompetenz. Dies mag zum einen daran liegen, dass in dieser frequenzanalytischen Auswertung qualitative Unterschiede in der dargestellten Technikkompetenz nicht berücksichtigt werden. Es kann natürlich auch an dem aktiven Versuch liegen, die dargestellten Personas bewusst nicht defizitorientiert zu beschreiben bzw. beide Geschlechter als kompetent darzustellen. Diese Interpretation ist naheliegend, da es sich hier zu einem großen Teil um Personas aus öffentlich geförderten Projekten handelt, in denen die Gleichstellung der Geschlechter eine explizite Anforderung darstellt.

Von den beiden zentralen Dimensionen der Globalgeschlechterstereotype sind Unterschiede in der Dimension Wärme/Gemeinschaft also stärker zu beobachten ist als in der Dimension Kompetenz/Instrumentalität. Bei der Annahme, dass bei den Personas versucht wurde, im Sinne der wahrgenommenen sozialen Erwünschtheit ,gute“ und damit eben möglichst keine geschlechterstereotypen Persona-Beschreibungen zu verfassen, kann dies folgendermaßen interpretiert werden: Während das Weniger-Vorhandensein von Wärme/Gemeinschaft bei Männern als eher unproblematisch gilt (und deshalb keine Notwendigkeit gesehen wird, hier in der Beschreibung von Personas korrigierend einzugreifen), stellt das Fehlen hinsichtlich der Dimension Kompetenz/Instrumentalität bei Frauen ein spürbares Defizit dar, welches im 
Zuge der Beschreibung von Personas einer Korrektur bedarf. Diese Interpretation ist konform mit Erkenntnissen zur gesamtgesellschaftlichen Veränderungen, wonach sich die von Frauen über sich selbst berichtete Instrumentalität kontinuierlich erhöht, die selbstberichtete Expressivität von Männern gleichzeitig jedoch unverändert bleibt (Twenge, 1997, 2009).

Insgesamt deuten die Daten somit darauf hin, dass die Personas daran orientiert sind, den gesellschaftlichen Status Quo abzubilden. Hier zeigt sich in westlichen Kulturen mehr und mehr die Tendenz, Eigenschaften und Verhalten aus dem Bereich Kompetenz/Instrumentalität weiter aufzuwerten und aus dem Bereich Wärme/Gemeinschaft abzuwerten. Twenge (2009) weist auf das darin liegende Paradox hin, dass darin besteht, dass der Status von Frauen sich erhöht hat während die ihnen traditionell zugeschriebenen Eigenschaften von Wärme/Gemeinschaft an Wert verloren haben. Sie empfiehlt auf dieser Basis, dass ein Empowerment von Mädchen und Frauen weniger Kompetenz/Instrumentalität bei Frauen betonen sollte als dass beiden Geschlechtern der Wert der Eigenschaften auf der Dimension Wärme/Gemeinschaft verdeutlicht wird.

\section{Implikationen für den Gestaltungsprozess}

Die für die untersuchten Persona-Beschreibungen gefundenen Hinweise auf Geschlechterstereotype zeigen, dass sich hier der gesellschaftliche Status Quo abbildet und das in der Gestaltung von HCI liegenden Potenzial zur aktiven Gestaltung des Wechselspiels von gesellschaftlicher und technischer Entwicklung nicht ausgeschöpft wird. Dies ist umso instruktiver, als dass sich Personas als geeignetes Mittel gezeigt haben, um bestehende Geschlechterstereotype zu hinterfragen.

Um dieses Potenzial im Gestaltungprozess zu nutzen, sollten Personas als Möglichkeit begriffen werden, um Genderaspekte in den menschzentrierten Gestaltungsprozess zu integrieren bzw. das Fortschreiben bestehender Geschlechterstereotype zu reflektieren. Da Stereotype den Default unserer Personenwahrnehmung darstellen, muss hier aktiv gegengesteuert werden, um das Potenzial der Persona-Methode in diese Richtung zu nutzen (Marsden et al., 2014), zum Beispiel indem Persona-Sets in persönlicher Ansprache formuliert werden oder eine zufällige Verteilung des Geschlechts vorgenommen wird.

\section{Literaturverzeichnis}

Allhutter, Doris (2012). Mind Scripting: A Method for Deconstructive Design. Science, Technology \& Human Values, 37(6), 684-707.

Banaji, Mahzarin \& Hardin, Curtis D. (1996). Automatic stereotyping. Psychological Science, 7(3), 136-141.

Bardzell, Shaowen \& Churchill, Elizabeth F. (2011). IwC Special Issue "Feminism and HCI: New Perspectives”. Special Issue Editors' Introduction. Interacting with Computers, 23(5), iii-xi.

Bath, Corinna (2014a). Diffractive Design. In N. Marsden \& U. Kempf (Eds.), Gender-UseIT - HCI, Usability und UX unter Gendergesichtspunkten (pp. 27-36). München: De Gruyter Oldenbourg. 
Bath, Corinna (2014b). Searching for Methodology. Feminist Technology Design in Computer Science. In Waltraud Ernst \& Ilona Horwath (Eds.), Gender in Science and Technology (pp. 57-78). Bielefeld: transcript.

Breslin, Samantha \& Wadhwa, Bimlesh (2015). Towards a Gender HCI Curriculum. Paper presented at the Proceedings of the 33rd Annual ACM Conference Extended Abstracts on Human Factors in Computing Systems, Seoul, Republic of Korea.

Buchmüller, Sandra, Joost, Gesche, Bessing, Nina \& Stein, Stephanie (2011). Bridging the gender and generation gap by ICT applying a participatory design process. Personal and Ubiquitous Computing, 15(7), 743-758.

Burnett, Margaret, Stumpf, Simone, MacBeth, James, Beckwith, Laura, Makri, Stephann, Kwan, Irwin \& Peters, Anicia (2014). Using the GenderMaP Method to Find Usability Issues through a Gender Lens. Retrieved Jan. 18th, 2015, from EUSES Consortium http://eusesconsortium.org/gender

Collins, Rebecca L. (2011). Content Analysis of Gender Roles in Media: Where Are We Now and Where Should We Go? Sex Roles, 64(3-4), 290-298.

Cuddy, Amy JC, Fiske, Susan T. \& Glick, Peter (2004). When professionals become mothers, warmth doesn't cut the ice. Journal of Social Issues, 60(4), 701-718.

Draude, Claude, Maaß, Susanne \& Wajda, Kamila (2014). GERD: ein Vorgehensmodell zur Integration von Gender/Diversity in die Informatik. In Anja Zeising, Claude Draude, Heidi Schelhowe \& Susanne Maßß (Eds.), Vielfalt der Informatik - Ein Beitrag zu Selbstverständnis und Außenwirkung (pp. 197-283). Bremen: Staats- und Universitätsbibliothek Bremen.

Eagly, Alice H, Wood, Wendy \& Diekman, Amanda B. (2000). Social role theory of sex differences and similarities: A current appraisal. The developmental social psychology of gender, 123-174.

Ebert, Irena, Steffens, Melanie \& Kroth, Alexandra (2014). Warm, but Maybe Not So Competent?Contemporary Implicit Stereotypes of Women and Men in Germany. Sex Roles, 70(9-10), 359-375.

Eckes, Thomas (2002). Paternalistic and envious gender stereotypes: Testing predictions from the stereotype content model. Sex Roles, 47(3-4), 99-114.

Eckes, Thomas (2008). Geschlechterstereotype: Von Rollen, Identitäten und Vorurteilen. In R. Becker \& B. Kortendiek (Eds.), Handbuch Frauen- und Geschlechterforschung (pp. 171-182): VS Verlag.

Ernst, Waltraud. (2014). Diffraction Patterns? Shifting Gender Norms in Biology and Technology. In Waltraud Ernst \& Ilona Horwath (Eds.), Gender in Science and Technology (pp. 147-163). Bielefeld: transcript Verlag.

Fiske, Susan T, Cuddy, Amy JC \& Glick, Peter (2007). Universal dimensions of social cognition: Warmth and competence. Trends in cognitive sciences, 11(2), 77-83.

Fiske, Susan T, Cuddy, Amy JC, Glick, Peter \& Xu, Jun (2002). A model of (often mixed) stereotype content: competence and warmth respectively follow from perceived status and competition. Journal of personality and social psychology, 82(6), 878-902.

Früh, Werner (2011). Inhaltsanalyse: Theorie und Praxis (Vol. 2501). UTB.

Greenwald, Anthony G, McGhee, Debbie E. \& Schwartz, Jordan LK. (1998). Measuring individual differences in implicit cognition: the implicit association test. Journal of personality and social psychology, 74(6), 1464-1480. 
Grudin, Jonathan (2006). Why personas work: The psychological evidence. In J. Pruitt \& T. Adlin (Eds.), The Persona Lifecycle (pp. 642-663). Amsterdam: Elsevier.

Grudin, Jonathan \& Williams, Gayna (2013). Two women who pioneered user-centered design. interactions, 20(6), 15-20.

Holtzblatt, Karen \& Beyer, Hugh (2015). Contextual Design Evolved. Morgan \& Claypool.

Källhammer, Eva \& Nilsson, Åsa Wikberg (2012). Gendered Innovative Design - Critical Reflections stimulated by Personas. In S. Andersson, K. Berglund, E. Gunnarsson \& E. Sundin (Eds.), Promoting Innovation - Policies, practices and procedures (pp. 328-350). Sweden: VINNOVA.

Kite, Mary E, Deaux, Kay \& Haines, Elizabeth L. (2008). Gender stereotypes. Psychology of women: A handbook of issues and theories, 2, 205-236.

Marsden, Nicola \& Kempf, Ute (2014). Gender-UseIT - HCI, Usability und UX unter Gendergesichtspunkten. München: De Gruyter Oldenbourg.

Marsden, Nicola, Link, Jasmin \& Büllesfeld, Elisabeth (2014). Personas und stereotype Geschlechterrollen. In N. Marsden \& U. Kempf (Eds.), Gender-UseIT - HCI, Usability und UX unter Gendergesichtspunkten (pp. 91-104). München: De Gruyter Oldenbourg.

Nielsen, Lene (2013). Personas - User Focused Design. Springer.

Oudshoorn, Nelly, Rommes, Els \& Stienstra, Marcelle (2004). Configuring the User as Everybody. Science, Technology \& Human Values, 29(1), 30-63.

Paulitz, Tanja \& Prietl, Bianca (2014). Geschlechter- und intersektionalitätskritische Perspektiven auf Konzepte der Softwaregestaltung. In Nicola Marsden \& Ute Kempf (Eds.), Gender-UseIT - HCI, Usability und UX unter Gendergesichtspunkten (pp. 79-89). München: De Gruyter Oldenbourg.

Pruitt, John \& Adlin, Tamara (2006). The Persona Lifecycle: Keeping People in Mind throughout Product Design. San Francisco: Morgan Kaufmann Publishers.

Ritter, Frank E., Baxter, Gordon D. \& Churchill, Elizabeth F. (2014). Foundations for Designing UserCentered Systems. Springer London.

Rommes, Els (2014). Feminist Interventions in the Design Process. In Waltraud Ernst \& Ilona Horwath (Eds.), Gender in Science and Technology (pp. 41-55). Bielefeld: transcript Verlag.

Rudy, Rena, Popova, Lucy \& Linz, Daniel (2011). Contributions to the Content Analysis of Gender Roles: An Introduction to a Special Issue. Sex Roles, 64(3-4), 151-159.

Swim, Janet K. \& Campbell, Bernadette. (2003). Sexism: Attitudes, beliefs, and behaviors. Blackwell handbook of social psychology: Intergroup processes, 218-237.

Turner, Phil \& Turner, Susan (2011). Is stereotyping inevitable when designing with personas? Design Studies, 32(1), 30-44.

Twenge, Jean M. (1997). Changes in masculine and feminine traits over time: A meta-analysis. Sex roles, 36(5-6), 305-325.

Twenge, Jean M. (2009). Status and gender: The paradox of progress in an age of narcissism. Sex Roles, 61(5-6), 338-340. 